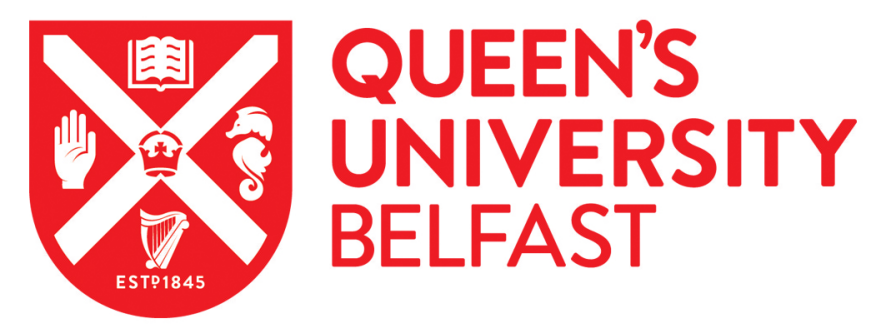

\title{
Sets of p-multiplicity in locally compact groups
}

Todorov, I. G., \& Turowska, L. (2015). Sets of p-multiplicity in locally compact groups. Studia Mathematica, 226 , 75-93. https://doi.org/10.4064/sm226-1-4

\author{
Published in: \\ Studia Mathematica
}

Document Version:

Peer reviewed version

Queen's University Belfast - Research Portal:

Link to publication record in Queen's University Belfast Research Portal

Publisher rights

(c) 2015 IMPAN

\section{General rights}

Copyright for the publications made accessible via the Queen's University Belfast Research Portal is retained by the author(s) and / or other copyright owners and it is a condition of accessing these publications that users recognise and abide by the legal requirements associated with these rights.

Take down policy

The Research Portal is Queen's institutional repository that provides access to Queen's research output. Every effort has been made to ensure that content in the Research Portal does not infringe any person's rights, or applicable UK laws. If you discover content in the Research Portal that you believe breaches copyright or violates any law, please contact openaccess@qub.ac.uk. 


\title{
SETS OF $p$-MULTIPLICITY IN LOCALLY COMPACT GROUPS
}

\author{
I. G. TODOROV AND L. TUROWSKA
}

\begin{abstract}
We initiate the study of sets of $p$-multiplicity in locally compact groups and their operator versions. We show that a closed subset $E$ of a second countable locally compact group $G$ is a set of $p$-multiplicity if and only if the set $E^{*}=\left\{(s, t): t s^{-1} \in E\right\}$ is a set of operator $p$-multiplicity. We exhibit examples of sets of $p$-multiplicity, establish preservation properties for unions and direct products, and prove a $p$ version of the Stone-von Neumann Theorem.
\end{abstract}

\section{INTRODUCTION}

The existence of non-zero compact operators acting on a Hilbert space and leaving invariant a given commutative subspace lattice was first examined in [10] (see also [6] and the references therein). That work followed W. B. Arveson's seminal paper [1], and showed that the presence of non-zero compact operators in CSL algebras is closely related to the notion of multiplicity sets in commutative Harmonic Analysis. This relation was formalised, and generalised to non-commutative locally compact groups, in [17], where the notion of sets of operator multiplicity was introduced, and [18], where it was shown that a closed subset $E$ of a (second countable) locally compact group $G$ is a set of multiplicity if and only if the set $E^{*}=\left\{(s, t): t s^{-1} \in E\right\}$ is a set of operator multiplicity.

The study of non-zero operators from Schatten $p$-classes in CSL algebras was also initiated in [10], where a link between such operators and pseudomeasures on compact abelian groups, whose Fourier transforms belong to the sequence space $\ell^{p}$, was exhibited. In the case $\ell^{p}$ is replaced by $c_{0}$, this turns into a special case of the result described in the previous paragraph. It is thus natural to define and study sets of $p$-multiplicity, their operator analogues, and the relation between these two notions.

This is the aim of the present article. In Section 3, given a locally compact group $G$, we define a subspace $\mathcal{S}_{p}(G)$ of the reduced group C*-algebra $C_{r}^{*}(G)$ of $G$ that plays a role analogous to the role of the Schatten $p$-class within the $\mathrm{C}^{*}$-algebra of all compact operators on a Hilbert space. In the case the group $G$ is compact, the space $\mathcal{S}_{p}(G)$ coincides with the intersection of $C_{r}^{*}(G)$ with the Schatten $p$-class on $L^{2}(G)$. It should be noted that if $G$ is discrete, $\mathcal{S}_{p}(G)$ is equal to $C_{r}^{*}(G)$, and thus the interest in our work lies in the case where $G$ is locally compact and non-discrete; for example, in the case where $G$ is connected. After defining sets of $p$-multiplicity and their

Date: 18 December 2014.

2010 Mathematics Subject Classification. Primary 47L05; Secondary 43A46, 22 D25. 
operator versions, we show that a closed set $E \subseteq G$ is a set of $p$-multiplicity if and only if $E^{*}$ is a set of operator $p$-multiplicity. We give a number of examples of sets of $p$-multiplicity, and establish preservation properties for unions and direct products. We include characterisations of the sets of $p$ multiplicity in the case $p=1$ and $p=2$.

In Section 4, we prove a $p$-version of the Stone-von Neumann Theorem. Recall that this result can be stated by saying that the $\mathrm{C}^{*}$-algebra of all compact operators on $L^{2}(G)$ is generated by $C_{r}^{*}(G)$ and the multiplication algebra of the space $C_{0}(G)$ of all continuous functions on $G$ vanishing at infinity. Here, we obtain an analogous result for the Schatten $p$-class, using the space $\mathcal{S}_{p}(G)$ in the place of $C_{r}^{*}(G)$.

In Section 5, using the Fourier theory of compact groups, we give a different proof of the aforementioned transference theorem for sets of $p$ multiplicity, which we believe is interesting in its own right.

Finally, in Section 2 we collect the necessary background material and set notation.

\section{Preliminaries}

Let $(X, \mu)$ and $(Y, \nu)$ be standard $(\sigma$-finite) measure spaces. A subset $E \subseteq X \times Y$ is called marginally null if $E \subseteq(M \times Y) \cup(X \times N)$, where $M \subseteq X$ and $N \subseteq Y$ are null sets. Let $T(X, Y)$ be the projective tensor product $L^{2}(X) \hat{\otimes} L^{2}(Y)$. Every element $h \in T(X, Y)$ can be written as a series

$$
h=\sum_{i=1}^{\infty} f_{i} \otimes g_{i}, \quad f_{i} \in L^{2}(X), g_{i} \in L^{2}(Y), i \in \mathbb{N},
$$

where $\sum_{i=1}^{\infty}\left\|f_{i}\right\|_{2}^{2}<\infty$ and $\sum_{i=1}^{\infty}\left\|g_{i}\right\|_{2}^{2}<\infty$. Such an element $h$ may be considered either as a function $h: X \times Y \rightarrow \mathbb{C}$, defined up to a marginally null set and given by

$$
h(x, y)=\sum_{i=1}^{\infty} f_{i}(x) g_{i}(y)
$$

or as an element of the predual of the space $\mathcal{B}\left(L^{2}(X), L^{2}(Y)\right)$ of all bounded linear operators from $L^{2}(X)$ into $L^{2}(Y)$ via the pairing

$$
\langle T, h\rangle:=\sum_{i=1}^{\infty}\left(T f_{i}, \bar{g}_{i}\right) .
$$

We denote by $\|h\|_{T}$ the norm of $h \in T(X, Y)$.

Let $\mathfrak{S}(X, Y)$ be the multiplier algebra of $T(X, Y)$; by definition, a measurable function $w: X \times Y \rightarrow \mathbb{C}$ belongs to $\mathfrak{S}(X, Y)$ if the map $m_{w}: h \rightarrow w h$ leaves $T(X, Y)$ invariant, that is, if $w h$ coincides almost everywhere with a function from $T(X, Y)$, for every $h \in T(X, Y)$. The elements of $\mathfrak{S}(X, Y)$ are called (measurable) Schur multipliers; we refer the reader to [15] for relevant details. If $w \in \mathfrak{S}(X, Y)$, the adjoint of $m_{w}$, acting on $\mathcal{B}\left(L^{2}(X), L^{2}(Y)\right)$, will be denoted by $S_{w}$.

Throughout the paper, $G$ is a locally compact group. The Lebesgue spaces $L^{p}(G), p=1,2, \infty$, are with respect to left Haar measure $m ; d m(x)$ 
is shortened to $d x$ and the modular function of $G$ is denoted by $\Delta$. Let $\lambda: G \rightarrow \mathcal{B}\left(L^{2}(G)\right), s \mapsto \lambda_{s}$, be the left regular representation. The symbol $\lambda$ is used also for the corresponding representation of $L^{1}(G)$ on $L^{2}(G)$; thus, if $f \in L^{1}(G)$ then $\lambda(f)$ is the operator on $L^{2}(G)$ given by $\lambda(f)(g)=f * g$.

The reduced group $C^{*}$-algebra $C_{r}^{*}(G)$ of $G$ is the operator norm closure of $\left\{\lambda(f): f \in L^{1}(G)\right\}$, while the group von Neumann algebra of $G$ is its weak* closure. The Fourier algebra $A(G)$ of $G$ is the (commutative, regular, semi-simple) Banach algebra consisting of all complex functions $u$ on $G$ of the form

$$
x \rightarrow u(x)=\left(\lambda_{x} \xi, \eta\right),
$$

where $\xi, \eta \in L^{2}(G)$. The norm of an element $u \in A(G)$ is by definition the infimum of the products $\|\xi\|\|\eta\|$, where $\xi$ and $\eta$ are functions from $L^{2}(G)$ for which (2.1) holds. The Banach space dual of $A(G)$ can be canonically identified with $\operatorname{VN}(G)$ : for $T \in \mathrm{VN}(G)$ and $u$ as in (2.1), the pairing is given by

$$
\langle T, u\rangle=(T \xi, \eta)
$$

we refer the reader to [8] for this and further properties of $A(G)$.

We set $T(G)=T(G, G), \mathfrak{S}(G)=\mathfrak{S}(G, G)$ and $\mathcal{B}\left(L^{2}(G)\right)=\mathcal{B}\left(L^{2}(G)\right.$, $\left.L^{2}(G)\right)$. The map $P: T(G) \rightarrow A(G)$, given by

$$
P(f \otimes g)(t)=\left\langle\lambda_{t}, f \otimes g\right\rangle=\left(\lambda_{t} f, \bar{g}\right)=\int_{G} f\left(t^{-1} s\right) g(s) d s=g * \check{f}(t)
$$

(where $\check{f}(t)=f\left(t^{-1}\right)$ ) is the predual of the inclusion $\mathrm{VN}(G) \rightarrow \mathcal{B}\left(L^{2}(G)\right.$ ). Moreover, the following holds (see [18] for a proof):

Proposition 2.1. For every $h \in T(G)$, we have

$$
P(h)(t)=\int_{G} h\left(t^{-1} s, s\right) d s, \quad t \in G .
$$

Define

$$
N: L^{\infty}(G) \rightarrow L^{\infty}(G \times G) \text { by } N(f)(s, t)=f\left(t s^{-1}\right) .
$$

We will often use the fact that if $u \in A(G)$ then $N(u) \in \mathfrak{S}(G)$. More generally, the set of all continuous functions $u: G \rightarrow \mathbb{C}$ such that $N(u) \in$ $\mathfrak{S}(G)$ coincides with the algebra $M^{\mathrm{cb}} A(G)$ of all completely bounded, or Herz-Schur, multipliers of $A(G)$ [3], [19] (see also [11]). For $v \in A(G)$ and $T \in \mathrm{VN}(G)$, let $v \cdot T \in \mathrm{VN}(G)$ be the element of $\operatorname{VN}(G)$ given by

$$
\langle v \cdot T, u\rangle=\langle T, v u\rangle, \quad u \in A(G) ;
$$

we have $v \cdot T=S_{N(v)}(T)$ (see, e.g., [13]).

We denote by $\mathcal{S}_{p}(H)$ the Schatten $p$-class on a Hilbert space $H$ (here, $1 \leq p<\infty)$, and we let $S_{\infty}(H)$ be the space of all compact operators on $H$. If $H$ is clear from the context, we simply write $\mathcal{S}_{p}$. We write $\|T\|_{p}$ for the Schatten $p$-norm of an element $T \in \mathcal{S}_{p}, 1 \leq p<\infty$, and let $\|T\|_{\infty}=\|T\|$ for the usual operator norm of an element $T \in \mathcal{S}_{\infty}$. 
For a function $h \in L^{2}(G \times G)$, we let $T_{h} \in \mathcal{S}_{2}\left(L^{2}(G)\right)$ be the operator given by

$$
T_{h}(\xi)(y)=\int_{G} h(y, x) \xi(x) d x, \quad \xi \in L^{2}(G), y \in G .
$$

We call the function $h$ the integral kernel of $T_{h}$. We note that if $h \in T(G)$ then $T_{h} \in \mathcal{S}_{1}\left(L^{2}(G)\right)$; conversely, for every operator $T \in \mathcal{S}_{1}\left(L^{2}(G)\right)$ there exists $h \in T(G)$ such that $T=T_{h}$.

For a measure space $(X, \mu)$, and a function $a \in L^{\infty}(X, \mu)$, we let $M_{a}$ denote the (bounded) operator on $L^{2}(X)$ of multiplication by $a$ and $P_{K}$ the multiplication by the characteristic function $\chi_{K}$ of measurable subset $K \subseteq X$.

\section{Definitions And PROPERTies}

Let $G$ be a locally compact group. For each $1 \leq p \leq \infty$, let

$$
\mathcal{S}_{p}(G)=\left\{T \in C_{r}^{*}(G): P_{K} T P_{K} \in \mathcal{S}_{p} \text {, for all compact subsets } K \subseteq G\right\} .
$$

Note first that, if $f \in C_{c}(G)$ then $P_{K} \lambda(f) P_{K}$ is an integral operator with integral kernel

$$
(s, t) \mapsto \chi_{K \times K}(s, t) \Delta(t)^{-1} f\left(s t^{-1}\right) .
$$

Thus, $P_{K} \lambda(f) P_{K}$ is a Hilbert-Schmidt operator. Since every $T \in C_{r}^{*}(G)$ can be approximated in the operator norm by operators of the form $\lambda(f)$ with $f \in C_{c}(G)$, we conclude that $P_{K} T P_{K} \in \mathcal{S}_{\infty}$ whenever $T \in C_{r}^{*}(G)$ and $K \subseteq G$ is compact; thus, $\mathcal{S}_{\infty}(G)=C_{r}^{*}(G)$.

Remarks (i) Let $v \in M^{\mathrm{cb}} A(G)$ and $T \in \mathcal{S}_{p}(G)$. Then $v \cdot T \in \mathcal{S}_{p}(G)$. Indeed, for every compact set $K \subseteq G$, we have that

$$
P_{K}(v \cdot T) P_{K}=P_{K} S_{N(v)}(T) P_{K}=S_{N(v)}\left(P_{K} T P_{K}\right) \in \mathcal{S}_{p},
$$

since Schur multipliers leave $\mathcal{S}_{p}$ invariant (the latter fact can be easily seen by using a complex interpolation argument, see $[2,16]$ and the proof of Theorem 3.3).

(ii) If $p \leq q$ then $\mathcal{S}_{p}(G) \subseteq \mathcal{S}_{q}(G)$.

(iii) If $G$ is discrete, $K \subseteq G$ is compact precisely when it is finite; thus, in this case, $\mathcal{S}_{p}(G)=C_{r}^{*}(G)$ for all values of $p$.

(iv) If $G$ is compact then $\mathcal{S}_{p}(G)=C_{r}^{*}(G) \cap \mathcal{S}_{p}$. Indeed, the inclusion $\mathcal{S}_{p} \cap C_{r}^{*}(G) \subseteq \mathcal{S}_{p}(G)$ holds trivially for any $G$. If $G$ is compact and $T \in \mathcal{S}_{p}(G)$ then, taking $K=G$ in the definition of $\mathcal{S}_{p}(G)$, we see that $T \in \mathcal{S}_{p}$.

In case $G$ is compact, the previous paragraph shows that $\mathcal{S}_{p}(G)$ is an ideal of $C_{r}^{*}(G)$. Moreover, the inclusion $S_{p}(G) \subseteq S_{q}(G), p<q$, is proper if $G$ is infinite (see Remark 5.2). We do not know whether the spaces $\mathcal{S}_{p}(G)$ are ideals for other classes of locally compact groups $G$.

(v) The identity from Remark (iv) fails when $G$ is not compact. Indeed, it is known (see, e.g., [20]) that in this case $\operatorname{VN}(G) \cap \mathcal{S}_{\infty}=\{0\}$.

(vi) Since for any compact subset $K \subseteq G$ and $f \in C_{c}(G)$ the operator $P_{K} \lambda(f) P_{K}$ is Hilbert-Schmidt, $\lambda\left(C_{c}(G)\right) \subseteq \mathcal{S}_{2}(G)$. 
(vii) Let $G$ be a compact abelian group and $\widehat{G}$ be its dual group. Then $\mathrm{VN}(G)$ and $C_{r}^{*}(G)$ can be identified, via Fourier transform, with the spaces $\ell^{\infty}(\widehat{G})$ and $c_{0}(\widehat{G})$. It is easily seen that, under this identification, $\mathcal{S}_{p}(G)$ is sent onto the sequence space $\ell_{p}(\widehat{G})$. Thus, in this case we have $\mathcal{S}_{p}(G)=$ $\mathrm{VN}(G) \cap \mathcal{S}_{p}=C_{r}^{*}(G) \cap \mathcal{S}_{p}$.

(viii) Let $G=\mathbb{R}$ and $L \subseteq \widehat{\mathbb{R}}=\mathbb{R}$ be a compact interval. Then $\chi_{L} \in$ $L^{\infty}(\widehat{\mathbb{R}}) \backslash C_{0}(\hat{\mathbb{R}})$. Thus, if $T$ is the operator in $\mathrm{VN}(\mathbb{R})$ corresponding to $\chi_{L}$ via Fourier transform, then $T \notin C_{r}^{*}(\mathbb{R})$. However, if $K \subseteq \mathbb{R}$ is compact then the operator $P_{K} T P_{K}$ is easily seen to be an integral operator with integral kernel

$$
(s, t) \mapsto \chi_{K \times K}(s, t) \int_{L} e^{-i x(s-t)} d x .
$$

Since $\left|\int_{L} e^{-i x(s-t)} d x\right| \leq m(L)$ for all $s, t \in \mathbb{R}$, the operator $P_{K} T P_{K}$ belongs to $\mathcal{S}_{2}$. This example shows that, in contrast to the compact case, replacing $C_{r}^{*}(G)$ by $\operatorname{VN}(G)$ in the definition of $\mathcal{S}_{p}(G)$ will in general yield different spaces.

Recall that, given a closed subset $E \subseteq G, I(E)$ (resp. $J(E)$ ) is the largest (resp. the smallest) ideal of $A(G)$ with null set $E$ :

$$
I(E)=\{u \in A(G): u(s)=0, s \in E\}
$$

and

$$
J(E)=\overline{\{u \in A(G): u \text { has compact support disjoint from } E\}} .
$$

For $J \subseteq A(G)$ we denote by $J^{\perp}$ the annihilator of $J$ in $\operatorname{VN}(G)$.

Sets of multiplicity (or $M$-sets) in (general) locally compact groups were introduced in [4] (see also [5]), while in [18], the notion of $M_{1}$-set was defined. We next formulate $p$-versions of these concepts as follows.

Definition 3.1. A closed subset $E \subseteq G$ will be called

(i) an $M^{p}$-set (or a set of p-multiplicity) if $J(E)^{\perp} \cap \mathcal{S}_{p}(G) \neq\{0\}$;

(ii) an $M_{1}^{p}$-set if $I(E)^{\perp} \cap \mathcal{S}_{p}(G) \neq\{0\}$.

It is clear that every $M_{1}^{p}$-set is an $M^{p}$-set, and that $M_{1}^{\infty}$-sets (resp. $M^{\infty}$ sets) coincide precisely with $M_{1}$-sets (resp. $M$-sets) studied in [18].

Recall that the support $\operatorname{supp}(T)$ of an operator $T \in \mathrm{VN}(G)$ is defined by letting

$$
\operatorname{supp}(T)=\{t \in G: u \cdot T \neq 0 \text { whenever } u \in A(G) \text { and } u(t) \neq 0\} .
$$

Note that $J(E)^{\perp}$ coincides with the space of all operators $T \in \mathrm{VN}(G)$ for which $\operatorname{supp}(T) \subseteq E$. Hence a susbet $E$ is an $M^{p}$-set if and only if there exists a non-zero operator $T$ in $S_{p}(G)$ with $\operatorname{supp}(T) \subseteq E$.

Our next aim is to define operator versions of sets of $p$-multiplicity. We first recall some concepts from [1] and [7]. Given standard measure spaces $(X, \mu)$ and $(Y, \nu)$, a subset $E$ of $X \times Y$ is called $\omega$-open if it is marginally equivalent to the union of a countable set of Borel rectangles. The complements of $\omega$-open sets are called $\omega$-closed. A function $w: X \times Y \rightarrow \mathbb{C}$ is called $\omega$-continuous if $w^{-1}(U)$ is an $\omega$-open set for every open set $U \subseteq \mathbb{C}$. 
If $F \subseteq X \times Y$ is an $\omega$-closed set, an operator $T \in \mathcal{B}\left(L^{2}(X), L^{2}(Y)\right)$ is said to be supported on $F$ if

$$
(A \times B) \cap F \simeq \emptyset \Longrightarrow P_{B} T P_{A}=0,
$$

for all measurable rectangles $A \times B \subseteq X \times Y$. A masa-bimodule is a subspace $\mathcal{U} \subseteq \mathcal{B}\left(L^{2}(X), L^{2}(Y)\right)$ such that $\mathcal{D}_{Y} \mathcal{U} \mathcal{D}_{X} \subseteq \mathcal{U}$ (where $\mathcal{D}_{X}$ (resp. $\mathcal{D}_{Y}$ ) is the multiplication masa of $L^{\infty}(X)$ (resp. $L^{\infty}(Y)$ ). Given a masa-bimodule $\mathcal{U}$, there exists a smallest, up to marginal equivalence, $\omega$-closed subset $\kappa \subseteq$ $X \times Y$ such that every operator in $\mathcal{U}$ is supported by $F$; we call $F$ the support of $\mathcal{U}$. Given an $\omega$-closed set $\kappa \subseteq X \times Y$, there exist [1], [7] a largest weak ${ }^{*}$ closed masa-bimodule $\mathfrak{M}_{\max }(\kappa)$ and a smallest weak ${ }^{*}$ closed masabimodule $\mathfrak{M}_{\text {min }}(\kappa)$ with support $\kappa$. The masa-bimodule $\mathfrak{M}_{\text {max }}(\kappa)$ is the space of all $T \in \mathcal{B}\left(L^{2}(X), L^{2}(Y)\right)$ supported on $\kappa$.

Definition 3.2. An $\omega$-closed subset $\kappa \subseteq X \times Y$ will be called

(i) an operator $M^{p}$-set (or a set of operator p-multiplicity) if $\mathfrak{M}_{\max }(\kappa) \cap$ $\mathcal{S}_{p} \neq\{0\}$

(ii) an operator $M_{1}^{p}$-set if $\mathfrak{M}_{\min }(\kappa) \cap \mathcal{S}_{p} \neq\{0\}$.

Remarks (i) Note that, if $1 \leq p \leq 2$ then the two notions introduced in Definition 3.2 agree; this follows from the fact that every Hilbert-Schmidt operator is pseudo-integral, while the pseudo-integral operators supported on a subset $\kappa$ are contained (in fact, weak ${ }^{*}$ dense) in $\mathfrak{M}_{\min }(\kappa)$. We refer the reader to [1] for the definition and more details about the class of pseudointegral operators.

(ii) A subset $\kappa \subseteq X \times Y$ is an operator $M^{2}$-set if and only if $(\mu \times \nu)(\kappa)>$ 0 ; indeed, the latter condition is equivalent to the existence of non-zero functions $h \in L^{2}(X \times Y)$ supported on $\kappa$.

In [18] we established a connection between sets of multiplicity and sets of operator multiplicity. The next theorem is a generalisation of this result to sets of $p$-multiplicity. For $\varphi \in T(G)$, let $E_{\varphi}: \mathcal{B}\left(L^{2}(G)\right) \rightarrow V N(G)$ be the map given by

$$
\left\langle E_{\varphi}(T), u\right\rangle=\langle T, \varphi N(u)\rangle, \quad T \in \mathcal{B}\left(L^{2}(G)\right), u \in A(G),
$$

where the pairing on the left hand side is the one between $\operatorname{VN}(G)$ and $A(G)$, and on the right hand side - the one between $\mathcal{B}\left(L^{2}(G)\right)$ and $T(G)$. It was proved in [18, Theorem 3.8] that $E_{\varphi}(T) \in C_{r}^{*}(G)$ for any $\varphi \in T(G)$ whenever $T$ is compact.

For $E \subseteq G$, we let $E^{*}=\left\{(s, t): t s^{-1} \in E\right\} \subseteq G \times G$. We will assume, for the rest of the paper, that $G$ is second countable.

Theorem 3.3. Let $G$ be a locally compact group, $E \subseteq G$ be a closed subset and $p \geq 1$. The following are equivalent:

(i) $E$ is an $M^{p}$-set (resp. an $M_{1}^{p}$-set);

(ii) $E^{*}$ is an operator $M^{p}$-set (resp. an operator $M_{1}^{p}$-set).

Proof. (i) $\Rightarrow$ (ii) Suppose that $E \subseteq G$ is an $M^{p}$-set and let $T$ be a non-zero operator in $J(E)^{\perp} \cap \mathcal{S}_{p}(G)$. Then there exists a compact set $K \subseteq G$ such that $P_{K} T P_{K}$ is non-zero; by [18, Lemma 3.11], $P_{K} T P_{K} \in \mathfrak{M}_{\max }\left(E^{*}\right) \cap \mathcal{S}_{p}$. 
Let $E \subseteq G$ be an $M_{1}^{p}$-set. The proof of [18, Theorem $\left.3.12(\mathrm{~b})\right]$ shows that $I(E)^{\perp} \subseteq \mathfrak{M}_{\min }\left(E^{*}\right)$. As in the previous paragraph, one can find a non-zero operator in $\mathfrak{M}_{\min }\left(E^{*}\right) \cap \mathcal{S}_{p}$.

(ii) $\Rightarrow$ (i) Assume that $E^{*}$ is an operator $M^{p}$-set; we will show that $E$ is an $M^{p}$-set. If $p=\infty$, this follows from [18, Theorem 3.11]. Let $p=1$ and $T$ be a non-zero trace class operator in $\mathfrak{M}_{\max }\left(E^{*}\right)$; by virtue of $(2.3)$ and the remark following it, write $T=T_{h}$, where $h=\sum_{i=1}^{\infty} f_{i} \otimes g_{i}$, with $\sum_{i=1}^{\infty}\left\|f_{i}\right\|_{2}^{2}<\infty$ and $\sum_{i=1}^{\infty}\left\|g_{i}\right\|_{2}^{2}<\infty$

Fix $\varphi \in T(G) \cap \mathfrak{S}(G)$ such that the function $\psi=\varphi(1 \otimes \Delta)$ belongs to $\mathfrak{S}(G)$. We will show that $E_{\varphi}(T) \in \mathcal{S}_{1}(G)$. For every $u \in A(G)$, we have

$$
\begin{aligned}
\left\langle E_{\varphi}(T), u\right\rangle & =\langle T, \varphi N(u)\rangle=\iint_{G \times G} h(s, t) \varphi(s, t) u\left(t s^{-1}\right) d s d t \\
& =\iint_{G \times G} h\left(r^{-1} t, t\right) \varphi\left(r^{-1} t, t\right) u(r) \Delta\left(t r^{-1}\right) d r d t \\
& =\int_{G}\left(\int_{G} h\left(r^{-1} t, t\right) \varphi\left(r^{-1} t, t\right) \Delta(t) d t\right) u(r) \Delta\left(r^{-1}\right) d r .
\end{aligned}
$$

By assumption, $\psi \in \mathfrak{S}(G)$ and hence $\psi h \in T(G)$. By Proposition 2.1,

$$
P(\psi h)(r)=\int_{G} h\left(r^{-1} t, t\right) \varphi\left(r^{-1} t, t\right) \Delta(t) d t
$$

and hence

$$
\left\langle E_{\varphi}(T), u\right\rangle=\int_{G} P(\psi h)(r) u(r) \Delta\left(r^{-1}\right) d r .
$$

Let $\xi, \eta \in L^{2}(G)$ be such that $u(r)=\left(\lambda_{r}(\xi), \eta\right), r \in G$. Then, by (3.1),

$$
\begin{aligned}
\left(E_{\varphi}(T) \xi, \eta\right) & =\left\langle E_{\varphi}(T), u\right\rangle=\iint_{G \times G} P(\psi h)(r) \Delta\left(r^{-1}\right) \xi\left(r^{-1} x\right) \overline{\eta(x)} d r d x \\
& =\iint_{G \times G} P(\psi h)\left(x y^{-1}\right) \Delta\left(y x^{-1}\right) \xi(y) \overline{\eta(x)} \Delta\left(y^{-1}\right) d y d x \\
& =\iint_{G \times G} P(\psi h)\left(x y^{-1}\right) \Delta\left(x^{-1}\right) \xi(y) \overline{\eta(x)} d y d x .
\end{aligned}
$$

Let

$$
w(x, y)=P(\psi h)\left(x y^{-1}\right) \Delta\left(x^{-1}\right), \quad x, y \in G .
$$

Identity (3.2) shows that $w$ is an integral kernel and $E_{\varphi}(T)=T_{w}$. If $K \subseteq G$ is compact then $P_{K} T_{w} P_{K}=T_{w \chi_{K \times K}}$ and

$$
w \chi_{K \times K}=\hat{N}(P(\psi h))\left(\left(\Delta^{-1} \chi_{K}\right) \otimes \chi_{K}\right),
$$

where $\hat{N}(v)(s, t)=v\left(s t^{-1}\right), s, t \in G$. We have that $P(\psi h) \in A(G)$; thus $N(P(\psi h)) \in \mathfrak{S}(G)$ and hence $\hat{N}(P(\psi h)) \in \mathfrak{S}(G)$. Since $\left(\Delta^{-1} \chi_{K}\right) \otimes \chi_{K} \in$ $T(G)$, identity (3.4) shows that $w \chi_{K \times K} \in T(G)$ and hence $P_{K} E_{\varphi}(T) P_{K} \in$ $\mathcal{S}_{1}$. Thus, $E_{\varphi}(T) \in \mathcal{S}_{1}(G)$.

By [18, Lemma 3.10], there exist elements $c, d \in L^{2}(G)$ such that $E_{c \otimes d}(T)$ $\neq 0$. Since the space $\mathcal{F}$ of all compactly supported functions in $L^{\infty}(G)$ is dense in $L^{2}(G)$, the continuity of the map $\varphi \rightarrow E_{\varphi}(T)$ and [18, Proposition 
3.8] imply that we may choose $c$ and $d$ from $\mathcal{F}$. However, in this case $d \Delta \in$ $L^{\infty}(G)$ and hence $(c \otimes d)(1 \otimes \Delta) \in \mathfrak{S}(G)$. Letting $\varphi=c \otimes d$, we then have by the previous paragraphs that $E_{\varphi}(T) \neq 0$, and by the proof of $[18$, Theorem 3.11] that $E_{\varphi}(T) \in J(E)^{\perp}$. It follows that $E$ is an $M^{1}$-set.

To prove the statement for an arbitrary $p$, we use complex interpolation. Recall [16] that $\left(\mathcal{S}_{1}, \mathcal{S}_{\infty}\right)$ is a compatible couple, and $\mathcal{S}_{p}$ coincides with the interpolation space between $\mathcal{S}_{1}$ and $\mathcal{S}_{\infty}$ with parameter $\theta=p^{-1}$. Let, as above, $\varphi \in T(G) \cap \mathfrak{S}(G)$ be such that the function $\psi=\varphi(1 \otimes \Delta)$ is an element of $\mathfrak{S}(G)$. For a fixed compact set $K \subseteq G$ and $p=1, \infty$, let $\Phi_{p}$ : $\mathcal{S}_{p} \rightarrow \mathcal{B}\left(L^{2}(G)\right)$ be the operator given by

$$
\Phi_{p}(T)=P_{K} E_{\varphi}(T) P_{K}, \quad T \in \mathcal{S}_{p} .
$$

By the previous paragraphs, the image of $\Phi_{1}$ is in $\mathcal{S}_{1}$. Moreover,

$$
\begin{aligned}
\left\|\Phi_{1}(T)\right\|_{\mathcal{S}_{1}} & =\left\|\chi_{K \times K} w\right\|_{T(G)}=\left\|\hat{N}(P(\psi h))\left(\left(\Delta^{-1} \chi_{K}\right) \otimes \chi_{K}\right)\right\|_{T(G)} \\
& \leq\|\hat{N}(P(\psi h))\|_{\mathfrak{S}(G)}\left\|\left(\left(\Delta^{-1} \chi_{K}\right) \otimes \chi_{K}\right)\right\|_{T(G)} \\
& \leq\|P(\psi h)\|_{A(G)}\left\|\left(\left(\Delta^{-1} \chi_{K}\right) \otimes \chi_{K}\right)\right\|_{T(G)} \\
& \leq\|\psi h\|_{T(G)}\left\|\left(\left(\Delta^{-1} \chi_{K}\right) \otimes \chi_{K}\right)\right\|_{T(G)} \\
& \leq m(K)\|\psi\|_{\mathfrak{S}(G)}\|h\|_{T(G)}\left\|\Delta^{-1} \chi_{K}\right\|_{\infty} \\
& =m(K)\|\psi\|_{\mathfrak{S}(G)}\left\|\Delta^{-1} \chi_{K}\right\|_{\infty}\|T\|_{\mathcal{S}_{1}},
\end{aligned}
$$

which shows that the operator $\Phi_{1}: \mathcal{S}_{1} \rightarrow \mathcal{S}_{1}$ is bounded. On the other hand, the image of $\Phi_{\infty}$ is in $\mathcal{S}_{\infty}$ and, by [18, Theorem 3.8],

$$
\left\|\Phi_{\infty}(T)\right\| \leq\|\varphi\|_{T(G)}\|T\|, \quad T \in \mathcal{S}_{\infty} .
$$

By complex interpolation, the image of the operator $\Phi_{p}$ is in $\mathcal{S}_{p}$. The proof is now completed by choosing $\varphi$ for which $E_{\varphi}(T)$ is non-zero.

We have thus shown that if $E^{*}$ is an operator $M^{p}$-set then $E$ is an $M^{p}$ set. The proof of the case where $E^{*}$ is an operator $M_{1}^{p}$-set follows similar arguments and uses the fact that $E_{\varphi}(T)$ belongs to $I(E)^{\perp}$ if $T \in \mathfrak{M}_{\min }\left(E^{*}\right)$ (see [18, Theorem 3.11]).

Corollary 3.4. A closed subset $E$ of a locally compact group $G$ is an $M^{1}$-set if and only if it has a non-empty interior.

Proof. Suppose that $E$ is an $M^{1}$-set. By Theorem 3.3, $\mathfrak{M}_{\max }\left(E^{*}\right)$ contains a non-zero trace class operator; by $[7$, Theorem 6.7$], E^{*}$ contains a non-trivial measurable rectangle, say, $\alpha \times \beta$. Thus $\beta \alpha^{-1} \subseteq E$. By Steinhaus' Theorem, $\beta \alpha^{-1}$, and hence $E$, has a non-empty interior.

Conversely, assume that $U$ is an open subset of $E$; we may further assume that $U$ has a compact closure contained in $E$. Let $u \in A(G)$ be a function supported in $U$; then $u \in L^{1}(G)$ and thus $\lambda(u) \in C_{r}^{*}(G)$. It is easy to see that $\lambda(u) \in J(E)^{\perp}$. Let $K \subseteq G$ be a compact set. Then $P_{K} \lambda(u) P_{K}$ is an integral operator with integral kernel $(t, s) \rightarrow u\left(t s^{-1}\right) \chi_{K}(t) \chi_{K}(s) \Delta(s)^{-1}$. The function $(t, s) \mapsto \chi_{K}(t) \chi_{K}(s) \Delta(s)^{-1}$ belongs to $T(G)$ since $\chi_{K}$ is compactly supported and $\Delta$ is continuous. Since $N(u) \in \mathfrak{S}(G)$, we conclude that the function $(t, s) \mapsto u\left(t s^{-1}\right) \chi_{K}(t) \chi_{K}(s) \Delta(s)^{-1}$ belongs to $T(G)$; since this holds for all compact sets $K$, we conclude that $\lambda(u) \in \mathcal{S}_{1}(G)$. 
Corollary 3.5. A closed subset $E$ of a locally compact group $G$ is an $M^{2}$-set if and only if it has positive Haar measure.

Proof. Note that $m(E)>0$ if and only if $m \times m\left(E^{*}\right)>0$. The claim follows from Theorem 3.3 and Remark (ii) after Definition 3.2.

We next include some examples.

Examples (i) J. Froelich [10, p. 13-14] has shown that there exists a closed set $E \subseteq \mathbb{T}$ of Lebesgue measure zero which supports a non-zero measure $\mu$ whose Fourier transform vanishes at infinity but which does not support a non-zero pseudomeasure with Fourier transform in $\ell^{p}$. The set $E$ is an $M$-set that is not an $M^{p}$-set for any $p \geq 1$.

(ii) Let $\mathbb{T}$ be the group of the unit circle, realised additively as $\mathbb{R} / 2 \pi \mathbb{Z}$. We identify $\mathbb{T}^{n}$ with $[-\pi, \pi)^{n}$, and view the sphere $S^{n-1}=\left\{x \in \mathbb{R}^{n}\right.$ : $|x|=1\}$ as a subset of $\mathbb{T}^{n}$. Let $\mu$ be the normalised surface area measure of $S^{n-1}$. A direct calculation (see, e.g., [21, p.154]) shows that $\hat{\mu}(k)=$ $C|k|^{-(n-2) / 2} J_{(n-2) / 2}(|k|)$ for some constant $C$, where $\hat{\mu}$ is the Fourier transform of $\mu$ and $J_{(n-2) / 2}$ is a Bessel function. As $\left|J_{\nu}(r)\right| \leq C_{\nu} r^{-1 / 2}$ (see [9, Theorem 5.1]), for large enough $r>0$, we obtain $\hat{\mu}(k)=O\left(\frac{1}{|k|^{(n-1) / 2}}\right)$, as $|k| \rightarrow \infty$.

We have

$$
\begin{aligned}
\sum_{k \in \mathbb{Z}^{n}}|\hat{\mu}(k)|^{p} & \leq C \sum_{k \in \mathbb{Z}^{n},|k| \geq 1} \frac{1}{|k|^{p(n-1) / 2}} \leq C \int_{x \in \mathbb{R}^{n},|x| \geq 1} \frac{1}{|x|^{(n-1) p / 2}} d x \\
& =\int_{1}^{\infty} \frac{r^{n-1}}{r^{(n-1) p / 2}} d r .
\end{aligned}
$$

Therefore for $p>2$ and $n>1+\frac{2}{p-2}$, the sequence $\{\hat{\mu}(k)\}_{k \in \mathbb{Z}^{n}}$ belongs to $\ell^{p}\left(\mathbb{Z}^{n}\right)$ and hence $\lambda(\mu) \in \mathcal{S}_{p}\left(\mathbb{T}^{n}\right)$. As $\lambda(\mu) \in I\left(S^{n-1}\right)^{\perp}$, we conclude that $S^{n-1} \subseteq \mathbb{T}^{n}$ is an $M_{1}^{p}$-set for $p>2$ whenever $n>1+\frac{2}{p-2}$. Note that, by Corollary 3.5, $S^{n-1}, n>1$, is not an $M_{1}^{2}$-set since $S^{n-1}$ has zero Lebesgue measure (see Corollary 3.5).

(iii) There exists a closed set $E \subseteq \mathbb{T}$ of Lebesgue measure zero and a non-zero measure $\mu$ with $\operatorname{supp} \mu=E$ such that $\widehat{\mu} \in \ell^{p}$ for any $p>2$ (see [23, Theorem 10.12]). Hence $E$ is an $M_{1}^{p}$-set for all $p>2$ but not an $M^{2}$-set (see Corollary 3.5).

(iv) In [7, p. 579] an example is given of a set $E \subseteq \mathbb{T}$ and a function $f \in L^{2}(\mathbb{T})$ such that $\lambda(f)$ is supported in $E$ and $\lambda(f) \in \mathcal{S}_{p}$ for any $p>1$ but $\lambda(f) \notin \mathcal{S}_{1}$. Remark (iv) from the start of Section 3 implies that $E$ is an $M^{p}$-set for all $p>1$ but not an $M^{1}$-set.

Following the established terminology in the classical case, let us call a closed subset $E \subseteq G$ a set of $p$-uniqueness if it is not a set of $p$-multiplicity. In the remainder of this section, we apply Theorem 3.3 to establish some preservation results for sets of $p$-uniqueness and sets of $p$-multiplicity. 
Proposition 3.6. Let $G$ be a locally compact group, $1 \leq p \leq \infty$ and $E_{i} \subseteq G$ be a closed subset, $i=1,2$. Then $E_{1} \cup E_{2}$ is a set of p-uniqueness if and only if $E_{1}$ and $E_{2}$ are sets of p-uniqueness.

Proof. To see the "if" part of the statement, it suffices, by Theorem 3.3, to show that if $\mathfrak{M}_{\max }\left(E_{i}^{*}\right) \cap \mathcal{S}_{p}=\{0\}$ for $i=1,2$, then $\mathfrak{M}_{\max }\left(\left(E_{1} \cup E_{2}\right)^{*}\right) \cap \mathcal{S}_{p}=$ $\{0\}$.

Let $D_{i}=\mathfrak{M}_{\max }\left(E_{i}^{*}\right)_{\perp}, i=1,2$, and suppose that $T \in \mathfrak{M}_{\max }\left(\left(E_{1} \cup E_{2}\right)^{*}\right) \cap$ $\mathcal{S}_{p}$. For each $\theta_{i} \in D_{i} \cap \mathfrak{S}(G), i=1,2$, we have that $\theta_{1} \theta_{2} \in D_{1} \cap D_{2}$ and, since $D_{1} \cap D_{2}=\mathfrak{M}_{\max }\left(\left(E_{1} \cup E_{2}\right)^{*}\right)_{\perp}$, we conclude that

$$
\left\langle S_{\theta_{1}}(T), \theta_{2}\right\rangle=\left\langle T, \theta_{1} \theta_{2}\right\rangle=0 .
$$

However, Schur multipliers leave $\mathcal{S}_{p}$ invariant; since $D_{2} \cap \mathfrak{S}(G)$ is dense in $D_{2}$ and $E_{2}$ is a set of $p$-uniqueness, $S_{\theta_{1}}(T)=0$. Thus, $\left\langle T, \theta_{1}\right\rangle=0$ for all $\theta_{1} \in D_{1} \cap \mathfrak{S}(G)$. Now the density of $D_{1} \cap \mathfrak{S}(G)$ in $D_{1}$ and the fact that $E_{1}$ is a set of $p$-uniqueness imply that $T=0$.

The 'only if' part of the statement follows from the fact that any closed subset of a set of $p$-uniqueness is a set of $p$-uniqueness.

Proposition 3.7. Let $G_{i}$ be a locally compact group, $1 \leq p \leq \infty$ and $E_{i} \subseteq G_{i}$ be a closed set, $i=1,2$. If $E_{i}$ is an $M^{p}$-set (resp. $M_{1}^{p}$-set), $i=1,2$, then $E_{1} \times E_{2} \subseteq G_{1} \times G_{2}$ is an $M^{p}$-set (resp. $M_{1}^{p}$-set).

Proof. Let

$$
\rho: G_{1} \times G_{1} \times G_{2} \times G_{2} \rightarrow G_{1} \times G_{2} \times G_{1} \times G_{2}
$$

be the map given by $\rho\left(s_{1}, t_{1}, s_{2}, t_{2}\right)=\left(s_{1}, s_{2}, t_{1}, t_{2}\right)$. We have that $\left(E_{1} \times\right.$ $\left.E_{2}\right)^{*}=\rho\left(E_{1}^{*} \times E_{2}^{*}\right)$.

By Theorem 3.3, it suffices to show that $\rho\left(E_{1}^{*} \times E_{2}^{*}\right)$ is an operator $M^{p_{-}}$ set (resp. an operator $M_{1}^{p}$-set). Denoting by $\otimes$ the algebraic tensor product, we have

$$
\mathfrak{M}_{\max }\left(E_{1}^{*}\right) \otimes \mathfrak{M}_{\max }\left(E_{2}^{*}\right) \subseteq \mathfrak{M}_{\max }\left(\rho\left(E_{1}^{*} \times E_{2}^{*}\right)\right)
$$

and

$$
\mathfrak{M}_{\text {min }}\left(E_{1}^{*}\right) \otimes \mathfrak{M}_{\text {min }}\left(E_{2}^{*}\right) \subseteq \mathfrak{M}_{\text {min }}\left(\rho\left(E_{1}^{*} \times E_{2}^{*}\right)\right)
$$

(see [18]). It follows that if $T_{i} \in \mathfrak{M}_{\max }\left(E_{1}^{*}\right) \cap \mathcal{S}_{p}$ (resp. $S_{i} \in \mathfrak{M}_{\max }\left(E_{1}^{*}\right) \cap \mathcal{S}_{p}$ ), $i=1,2$, then $T_{1} \otimes T_{2}$ (resp. $\left.S_{1} \otimes S_{2}\right)$ is a non-zero operator in $\mathfrak{M}_{\max }\left(\rho\left(E_{1}^{*} \times\right.\right.$ $\left.\left.E_{2}^{*}\right)\right) \cap \mathcal{S}_{p}\left(\right.$ resp. $\left.\mathfrak{M}_{\min }\left(\rho\left(E_{1}^{*} \times E_{2}^{*}\right)\right) \cap \mathcal{S}_{p}\right)$.

We finish this section with explicit descriptions of the spaces $\mathcal{S}_{1}(G)$ and $\mathcal{S}_{2}(G)$. For the next lemma, recall that, if $G$ is compact then $\mathfrak{S}(G) \subseteq T(G)$ and hence $N(u) \in T(G)$ for every $u \in A(G)$.

Lemma 3.8. Let $G$ be a compact group. If $u \in A(G)$ then $T_{N(\breve{u})}=\lambda(u)$. 
Proof. If $\xi, \eta \in L^{2}(G)$ then, using the unimodulrity of $G$, we have

$$
\begin{aligned}
\left(T_{N(\check{u})} \xi, \eta\right) & =\iint_{G \times G} N(\check{u})(t, s) \xi(s) \overline{\eta(t)} d s d t \\
& =\iint_{G \times G} u\left(t s^{-1}\right) \xi(s) \overline{\eta(t)} d s d t \\
& =\iint_{G \times G} u(r) \xi\left(r^{-1} t\right) \overline{\eta(t)} d r d t=(\lambda(u)(\xi), \eta) .
\end{aligned}
$$

We say that a function $f$ belongs to $A(G)$ at the point $t \in G$ if there exists a neighbourhood $U$ of $t$ and a function $u \in A(G)$ such that $f(s)=u(s)$ for all $s \in U$. We let $A(G)^{\text {loc }}$ denote the set of all functions that belong to $A(G)$ at every point $t \in G$.

Lemma 3.9. Let $w: G \times G \rightarrow \mathbb{C}$ be a measurable (with respect to product measure) function, such that, for every $r \in G, w(x r, y r)=w(x, y)$ for marginally almost all $(x, y)$. Then there exists a measurable function $u$ : $G \rightarrow \mathbb{C}$ such that, up to a null set, $w=N(u)$.

Proof. Let $x \in G$, and $v_{x}: G \rightarrow \mathbb{C}$ be given by $v_{x}(s)=w(s, x s), s \in G$. For $r \in G$, the set

$$
\{(y, z) \in G \times G: w(y r, z r) \neq w(y, z)\}
$$

is marginally null. In particular,

$$
\Lambda_{x} \stackrel{\text { def }}{=}\{(s, x s) \in G \times G: w(s r, x s r) \neq w(s, x s)\}
$$

is marginally null. This easily implies that the set $\left\{s \in G:(s, x s) \in \Lambda_{x}\right\}$ is null and hence $v_{x}(s r)=v_{x}(s)$ for almost all $s$. Using arguments similar to [14, Lemma 3.2], one can prove that the function $f_{x}(s, r)=v_{x}(s r)$ is $m \times m$-measurable. For every $r \in G$, the set $\left\{s \in G: v_{x}(s r)=v_{x}(s)\right\}$ is null. By the Fubini theorem,

$$
\iint\left|v_{x}(s r)-v_{x}(s)\right| d s d r=\int\left(\int\left|v_{x}(s r)-v_{x}(s)\right| d s\right) d r=0,
$$

giving $v_{x}(s r)=v_{x}(s)$ for almost all pairs $(s, r)$. Thus there exists $s_{0} \in G$ such that $v_{x}\left(s_{0} r\right)=v_{x}\left(s_{0}\right)$ for almost all $r \in G$. Hence, there exists $u(x) \in \mathbb{C}$ such that $v_{x}(s)=u(x)$ for almost all $s \in G$.

The function $u: G \rightarrow \mathbb{C}$ is measurable as the composition of $w$ and the measurable functions $x \rightarrow(s, x s)$. Since the functions $w$ and $N(u)$ are equal almost everywhere on each set of the form $\{x\}^{*}$, applying the above arguments we have that $w=N(u)$ almost everywhere.

Theorem 3.10. Let $G$ be a locally compact group. Then

$$
\begin{gathered}
\mathcal{S}_{1}(G)=\left\{T \in C_{r}^{*}(G): \text { there exists } u \in A(G)^{\text {loc }}\right. \text { such that } \\
\left.P_{K} T P_{K}=T_{N(u)\left(\Delta^{-1} \otimes 1\right) \chi_{K \times K}} \text { for each compact } K \subseteq G\right\} .
\end{gathered}
$$

Moreover, if $G$ is compact then

$$
\mathcal{S}_{1}(G)=\{\lambda(u): u \in A(G)\} .
$$


Proof. We first show that $\mathcal{S}_{1}(G)$ is contained in the right hand side of (3.5). By assumption, for every compact set $K \subseteq G$ there exists a function $h_{K} \in$ $T(G)$ such that $P_{K} T P_{K}=T_{h_{K}}$. If $L \subseteq G$ is compact then

$$
\begin{aligned}
T_{h_{K}\left(\chi_{K \cap L} \otimes \chi_{K \cap L}\right)} & =P_{K \cap L} P_{K} T P_{K} P_{K \cap L}=P_{K \cap L} T P_{K \cap L}=T_{h_{K \cap L}} \\
& =P_{K \cap L} P_{L} T P_{L} P_{K \cap L}=T_{h_{L}\left(\chi_{K \cap L} \otimes \chi_{K \cap L}\right)} .
\end{aligned}
$$

Thus, $h_{K}\left(\chi_{K \cap L} \otimes \chi_{K \cap L}\right)=h_{L}\left(\chi_{K \cap L} \otimes \chi_{K \cap L}\right)$ almost everywhere. Since the functions $h_{K}\left(\chi_{K \cap L} \otimes \chi_{K \cap L}\right)$ and $h_{L}\left(\chi_{K \cap L} \otimes \chi_{K \cap L}\right)$ are $\omega$-continuous, [17, Lemma 2.2] implies that they are equal up to a marginally null set. Let $\left(K_{n}\right)_{n=1}^{\infty}$ be an increasing sequence of compact sets such that $G=\cup_{n=1}^{\infty} K_{n}$. Setting $h(s, t)=h_{K_{n}}(s, t)$ if $s, t \in K_{n}$, we obtain a function $h: G \times G \rightarrow \mathbb{C}$, defined up to a marginally null set, which has the property that $\left.h\right|_{K \times K}$ is marginally equivalent to $h_{K \times K}$ for every compact set $K \subseteq G$.

Let $L \subseteq G$ be a compact set and $\xi, \eta \in L^{2}(G)$ be supported on $L$. Fix $s \in G$ and let $M \subseteq G$ be a compact set containing both $L$ and $L s^{-1}$. Let $\rho: G \rightarrow \mathcal{B}\left(L^{2}(G)\right), s \mapsto \rho_{s}$, be the right regular representation given by $\rho_{s} \xi(x)=\sqrt{\Delta(s)} \xi(x s)$. Then

$$
\begin{aligned}
\left(T \rho_{s} \xi, \eta\right) & =\left(P_{M} T P_{M} \rho_{s} \xi, \eta\right)=\left(T_{h_{M}} \rho_{s} \xi, \eta\right) \\
& =\int_{G \times G} h(x, y) \xi(y s) \overline{\eta(x)} \sqrt{\Delta(s)} d x d y \\
& =\int_{G \times G} h\left(x, z s^{-1}\right) \xi(z) \overline{\eta(x)} \sqrt{\Delta\left(s^{-1}\right)} d x d z .
\end{aligned}
$$

On the other hand,

$$
\begin{aligned}
\left(\rho_{s} T \xi, \eta\right) & =\left(T \xi, \rho_{s^{-1}} \eta\right)=\left(P_{M} T P_{M} \xi, \rho_{s^{-1}} \eta\right)=\left(T_{h_{M}} \xi, \rho_{s^{-1}} \eta\right) \\
& =\int_{G \times G} h\left(x^{\prime}, z\right) \xi(z) \overline{\eta\left(x^{\prime} s^{-1}\right)} \sqrt{\Delta\left(s^{-1}\right)} d x^{\prime} d z \\
& =\int_{G \times G} h(x s, z) \xi(z) \overline{\eta(x)} \sqrt{\Delta(s)} d x d z .
\end{aligned}
$$

Since $T \in C_{r}^{*}(G)$, we have $T \rho_{s}=\rho_{s} T$, and hence $\sqrt{\Delta\left(s^{-1}\right)} h\left(x, z s^{-1}\right)=$ $\sqrt{\Delta(s)} h(x s, z)$ for marginally almost all $(x, z) \in L \times L$. Since this holds for every compact set $L$, we have that $\sqrt{\Delta\left(s^{-1}\right)} h\left(x, z s^{-1}\right)=\sqrt{\Delta(s)} h(x s, z)$ for marginally almost all $(x, z) \in G \times G$. Thus, $h(x, y)=\Delta(s) h(x s, y s)$ for marginally almost all $(x, y) \in G \times G$.

Let $\tilde{h}: G \times G \rightarrow \mathbb{C}$ be given by $\tilde{h}(x, y)=\Delta(x) h(x, y)$. If $s \in G$ then

$$
\tilde{h}(x s, y s)=\Delta(x s) h(x s, y s)=\Delta(x) \Delta(s) h(x s, y s)=\Delta(x) h(x, y)=\tilde{h}(x, y),
$$

for marginally almost all $(x, y)$. By Lemma 3.9, there exists a measurable function $u: G \rightarrow \mathbb{C}$ such that, up to a null set, $\tilde{h}=N(u)$. Thus, up to a null set, $h=\left(\Delta^{-1} \otimes 1\right) N(u)$.

Note that, for every compact set $K \subseteq G$, we have $h \chi_{K \times K} \in T(G)$ and $P_{K} T P_{K}=T_{h \chi_{K \times K}}$. Thus, it remains to show that $u \in A(G)^{\text {loc }}$. We have that $\tilde{h} \chi_{K \times K} \in T(G)$ for every compact set $K \subseteq G$. Since $T(G)$ consists of 
local Schur multipliers (see [17]), the $\sigma$-compactness of $G$ implies that $\tilde{h}$ is a local Schur multiplier. By [18, Theorem 8.2], $u \in A(G)^{\text {loc }}$.

To see that the right hand side of $(3.5)$ is contaied in $\mathcal{S}_{1}(G)$, note first that for any compact set $L$ and $u \in A(G)^{\text {loc }}$ there exists $v \in A(G)$ such that $u(t)=v(t)$ for any $t \in L$ (see the discussion before [18, Lemma 6.1]). Let $K$ be a compact set and let $v \in A(G)$ such that $u=v$ on $L=K K^{-1}$. Then $N(u) \chi_{K \times K}=N(v) \chi_{K \times K}$. Since $N(v)$ is a Schur multiplier and $\left(\Delta^{-1} \otimes\right.$ 1) $\chi_{K \times K} \in T(G)$, we have that $h(x, y):=N(u)(x, y) \Delta^{-1}(x) \chi_{K \times K}(x, y) \in$ $T(G)$.

If $G$ is compact then $G$ is unimodular and $A(G)=A(G)^{\text {loc }}$. By Lemma $3.8, T_{N(\check{u})}=\lambda(u)$, and the proof is complete.

The proof of the next proposition is similar to that of Theorem 3.10 and is omitted.

Proposition 3.11. Let $G$ be a locally compact group. The following are equivalent, for an operator $T \in C_{r}^{*}(G)$ :

(i) $T \in \mathcal{S}_{2}(G)$;

(ii) there exists a measurable function $u: G \rightarrow \mathbb{C}$ such that, for every compact set $K \subseteq G$, we have $N(u) \chi_{K \times K} \in L^{2}(G \times G)$ and $P_{K} T P_{K}=$ $T_{N(u)\left(\Delta^{-1} \otimes 1\right) \chi_{K \times K}}$.

\section{A $p$-Version of the Stone-Von Neumann Theorem}

The aim of this section is to establish the following $p$-version of the Stonevon Neumann Theorem ([22, Theorem 4.23]). We let $\mathcal{D}=\left\{M_{a}: a \in C_{0}(G)\right\}$.

Theorem 4.1. Let $G$ be a locally compact group and $1 \leq p \leq \infty$. Then the $\|\cdot\|_{p}$-closed $\mathcal{D}$-bimodule generated by $\mathcal{S}_{p}(G)$ coincides with $\mathcal{S}_{p}$.

Proof. For $p=\infty$, the statement reduces to the Stone-von Neumann Theorem. Fix $p$ with $1 \leq p<\infty$. Let

$$
\mathcal{S}_{p}^{\prime}(G)=\left\{T \in C_{r}^{*}(G): P_{K} T, T P_{K} \in \mathcal{S}_{p}, \text { for every compact set } K \subseteq G\right\}
$$

and

$$
\mathcal{U}_{p}=\overline{\operatorname{span}\left\{M_{a} T M_{b}: a, b \in C_{c}(G), T \in \mathcal{S}_{p}^{\prime}(G)\right\}^{\|\cdot\|_{p}} .}
$$

We note first that $\mathcal{S}_{p}^{\prime}(G) \subseteq \mathcal{S}_{p}(G)$ and, by the definition of $\mathcal{S}_{p}(G)$, we have

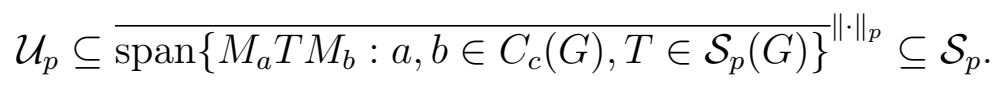

It follows from the proof of Theorem 3.10, that for $u \in A(G) \cap C_{c}(G)$, $P_{K} \lambda(u), \lambda(u) P_{K} \in \mathcal{S}_{1} \subseteq \mathcal{S}_{p}, p \geq 1$ and giving that both $\mathcal{S}_{p}^{\prime}(G)$ and $\mathcal{U}_{p}$ are non-zero.

We claim that $\mathcal{U}_{p}$ is an ideal of $\mathcal{S}_{\infty}$. First note that if $b \in C_{c}(G)$ and $f \in L^{1}(G)$ then by the Stone-von Neumann theorem $M_{b} \lambda(f)$ can be approximated in the operator norm by linear combinations $\sum_{i} \lambda\left(g_{i}^{n}\right) M_{c_{i}^{n}}$. Suppose that $T \in \mathcal{S}_{p}^{\prime}(G), S \in \mathcal{S}_{\infty}$ and $a, b, c \in C_{c}(G)$. Since $M_{a} T \in \mathcal{S}_{p}$ we have

$$
\left\|M_{a} T M_{b} \lambda(f)-M_{a} T \sum_{i} \lambda\left(g_{i}^{n}\right) M_{c_{i}^{n}}\right\|_{p} \leq\left\|M_{a} T\right\|_{p}\left\|M_{b} \lambda(f)-\sum_{i} \lambda\left(g_{i}^{n}\right) M_{c_{i}^{n}}\right\| \rightarrow 0
$$


and therefore $M_{a} T M_{b} \lambda(f) \in \mathcal{U}_{p}$. Again by the Stone-von-Neumann Theorem, $S$ can be approximated by linear combinations of operators of the form $\lambda(f) M_{c}$, where $f \in L^{1}(G)$ and $c \in C_{c}(G)$. Hence the corresponding linear combination of $M_{a} T M_{b} \lambda(f) M_{c}$ converges in $\|\cdot\|_{p}$-norm to $M_{a} T M_{b} S$. Thus, $M_{a} T M_{b} S \in \mathcal{U}_{p}$ and hence $\mathcal{U}_{p}$ is a right ideal of $\mathcal{S}_{\infty}$; one similarly shows that $\mathcal{U}_{p}$ is a left ideal in $\mathcal{S}_{\infty}$. Thus, $\mathcal{U}_{p}$ is a non-zero ideal of $\mathcal{S}_{\infty}$, closed in the Schatten $p$-norm. This easily implies that

$$
\mathcal{U}_{p}=\overline{\operatorname{span}\left\{M_{a} T M_{b}: a, b \in C_{c}(G), T \in \mathcal{S}_{p}(G)\right\}^{\|\cdot\|_{p}}}=\mathcal{S}_{p}
$$

\section{The CASE OF COMPACT GROUPS}

In this section, we include a direct proof of Theorem 3.3, that is, a proof that does not use interpolation, in the case $G$ is a compact group. We first recall some notions from the Fourier theory for compact groups (see, e.g, [12]).

Let $G$ be a compact group with dual $\widehat{G}$; thus, $\widehat{G}$ is a complete family of pairwise inequivalent continuous unitary representations $\pi: G \rightarrow \mathcal{B}\left(H_{\pi}\right)$ of $G$. We let $d_{\pi}=\operatorname{dim}\left(H_{\pi}\right)$. For $u \in A(G)$ we set

$$
\hat{u}(\pi)=\int_{G} u(s) \pi\left(s^{-1}\right) d s,
$$

understood as a linear operator on the finite-dimensional space $H_{\pi}$. Then

$$
\sum_{\pi \in \hat{G}} d_{\pi}\|\hat{u}(\pi)\|_{1}<\infty
$$

where $\|\cdot\|_{1}$ is the trace norm. Moreover, the Fourier algebra $A(G)$ can be identified with the space of operator fields, indexed over $\widehat{G}$,

$$
\left\{(f(\pi))_{\pi \in \widehat{G}}: f(\pi) \in \mathcal{B}\left(H_{\pi}\right), \sum_{\pi \in \hat{G}} d_{\pi}\|f(\pi)\|_{1}<\infty\right\},
$$

the identification being given by the map (which we call the Fourier transform) sending an element $u \in A(G)$ to $(\hat{u}(\pi))_{\pi \in \widehat{G}}$. Its inverse sends $(f(\pi))_{\pi \in \widehat{G}}$ to the funtion $f$ given by $f(s)=\sum_{\pi \in \widehat{G}} d_{\pi} \operatorname{Tr}(f(\pi) \pi(s)$ ) (where $\operatorname{Tr}$ denotes the trace). We can therefore identify the dual space of $A(G)$ with $\prod_{\pi \in \widehat{G}} \mathcal{B}\left(H_{\pi}\right)$ through the duality

$$
\left\langle\left(T_{\pi}\right)_{\pi \in \widehat{G}}, u\right\rangle=\sum_{\pi \in \widehat{G}} d_{\pi} \operatorname{Tr}\left(T_{\pi} \hat{u}(\pi)\right) .
$$

In particular, the evaluation functional at $s$ corresponds to $(\pi(s))_{\pi \in \widehat{G}}, s \in G$. The dual space of $A(G)$ is isomorphic to $\mathrm{VN}(G)$, and the identification of $\mathrm{VN}(G)$ with $\prod_{\pi \in \widehat{G}} \mathcal{B}\left(H_{\pi}\right)$ is given by $\left(T_{\pi}\right)_{\pi \in \widehat{G}} \mapsto \oplus_{\pi \in \widehat{G}} T_{\pi}^{\left(d_{\pi}\right)} \in \mathrm{VN}(G)$, where $T^{(k)}=\underbrace{T \oplus \cdots \oplus T}_{k}$. It follows from Remark (iv) at the start of Section 3 
that

$$
\mathcal{S}_{p}(G)=\left\{T \in C_{r}^{*}(G): \sum_{\pi \in \hat{G}} d_{\pi}\left\|T_{\pi}\right\|_{p}^{p}<\infty\right\} .
$$

Thus, $\mathcal{S}_{p}(G)$ is an ideal not only in $C_{r}^{*}(G)$ but also in $\operatorname{VN}(G)$.

Theorem 5.1. Let $G$ be a compact group, $p \geq 1$ and $E \subseteq G$ be a closed subset. The following are equivalent:

(i) $E$ is an $M^{p}$-set (resp. an $M_{1}^{p}$-set);

(ii) $E^{*}$ is an operator $M^{p}$-set (resp. an operator $M_{1}^{p}$-set).

Proof. (i) $\Rightarrow$ (ii) follows as in Theorem 3.3 .

(ii) $\Rightarrow$ (i) Suppose that $E^{*}$ is an operator $M^{p}$-set. If $T \in \mathfrak{M}_{\max }\left(E^{*}\right) \cap \mathcal{S}_{p}$ is non-zero, by [18, Lemma 3.10], there exist $a, b \in L^{\infty}(G)$ such that $E_{a \otimes b}(T) \in$ $C_{r}^{*}(G)$ is non-zero.

By the definition of the map $E_{a \otimes b}$ and Lemma 3.8, for $u \in A(G)$ we have

$$
\begin{aligned}
\left\langle E_{a \otimes b}(T), u\right\rangle & =\langle T, N(u)(a \otimes b)\rangle=\left\langle M_{b} T M_{a}, N(u)\right\rangle \\
& =\operatorname{Tr}\left(\left(M_{b} T M_{a}\right) T_{N(u)}\right)=\operatorname{Tr}\left(\left(M_{b} T M_{a}\right) \lambda(\check{u})\right) .
\end{aligned}
$$

Let $u \in A(G)$ be such that $\sum_{\pi \in \hat{G}} d_{\pi}\|\hat{u}(\pi)\|_{q}^{q}<\infty$, where $q$ is conjugate to $p$. The space of all such elements is dense in $A(G)$ (indeed, it contains all elements of $A(G)$ whose Fourier transform is finitely supported). By the the Peter-Weyl Theorem, $\lambda=\oplus_{\pi \in \hat{G}} \pi^{\left(d_{\pi}\right)}$, where $\pi^{\left(d_{\pi}\right)}=\underbrace{\pi \oplus \cdots \oplus \pi}_{d_{\pi}}$, and $\left.\lambda(\check{u})\right|_{H_{\pi}}=\hat{u}(\pi)$. Thus,

$$
\|\lambda(\check{u})\|_{q}^{q}=\sum_{\pi \in \hat{G}} d_{\pi}\|\hat{u}(\pi)\|_{q}^{q}<\infty .
$$

By (5.3) and (5.4),

$$
\left|\left\langle E_{a \otimes b}(T), u\right\rangle\right| \leq\|a\|_{\infty}\|b\|_{\infty}\|T\|_{p}\left(\sum_{\pi \in \hat{G}} d_{\pi}\|\hat{u}(\pi)\|_{q}^{q}\right)^{1 / q} .
$$

Let $S=E_{a \otimes b}(T)$. By $(5.1),\langle S, u\rangle=\sum_{\pi \in \hat{G}} d_{\pi} \operatorname{Tr}\left(S_{\pi} \hat{u}(\pi)\right)$. We claim that

$$
\sum_{\pi \in \hat{G}} d_{\pi}\left\|S_{\pi}\right\|_{p}^{p}<\infty
$$

In fact, let $S_{\pi}=V_{\pi}\left|S_{\pi}\right|$ be the polar decomposition of $S_{\pi}$. For any finite family $\mathcal{F} \subseteq \widehat{G}$, let $u \in A(G)$ be such that,

$$
\left.\hat{u}(\pi)=\left(\left|S_{\pi}\right|\right)^{p-1} V_{\pi}^{*}\right) /\left(\sum_{\pi \in \mathcal{F}} d_{\pi}\left\|S_{\pi}\right\|_{p}^{p}\right)^{(p-1) / p} \quad, \text { if } \pi \in \mathcal{F}
$$

and

$$
\hat{u}(\pi)=0 \text {, if } \pi \notin \mathcal{F} \text {. }
$$


We have

$$
\sum_{\pi \in \widehat{G}} d_{\pi}\|\hat{u}(\pi)\|_{q}^{q}=\sum_{\pi \in \mathcal{F}} d_{\pi} \operatorname{Tr}\left(\left|S_{\pi}\right|^{p}\right) / \sum_{\pi \in \mathcal{F}} d_{\pi}\left\|S_{\pi}\right\|_{p}^{p}=1
$$

and

$$
\begin{aligned}
\sum_{\pi \in \widehat{G}} d_{\pi} \operatorname{Tr}\left(S_{\pi} \hat{u}(\pi)\right) & =\sum_{\pi \in \mathcal{F}} d_{\pi} \operatorname{Tr}\left(\left|S_{\pi}\right|^{p}\right) /\left(\sum_{\pi \in \mathcal{F}} d_{\pi}\left\|S_{\pi}\right\|_{p}^{p}\right)^{(p-1) / p} \\
& =\left(\sum_{\pi \in \mathcal{F}} d_{\pi}\left\|S_{\pi}\right\|_{p}^{p}\right)^{1 / p} .
\end{aligned}
$$

Inequality (5.5) now implies $\left(\sum_{\pi \in \mathcal{F}} d_{\pi}\left\|S_{\pi}\right\| \|_{p}^{p}\right)^{1 / p} \leq\|a\|_{\infty}\|b\|_{\infty}\|T\|_{p}$, for any finite $\mathcal{F}$. Inequality (5.6) follows; by $(5.2), E_{a \otimes b}(T) \in \mathcal{S}_{p}(G)$. By the proof of [18, Theorem 3.11], $E_{a \otimes b}(T) \in J(E)^{\perp}$, and the proof is complete for $M^{p_{-}}$ sets. The proof of the statement for $M_{1}^{p}$-sets is similar and uses the fact that $E_{a \otimes b}(T) \in I(E)^{\perp}$ if $T \in \mathfrak{M}_{\min }\left(E^{*}\right)$.

Remark 5.2. If $G$ is a compact infinite group, then $\mathcal{S}_{p}(G)$ is a proper ideal of $\mathcal{S}_{q}(G)$ if $p<q$. In fact, one can easily find $\left\{\alpha_{\pi}\right\}_{\pi \in \hat{G}} \subseteq \mathbb{C}$ such that $\sum_{\pi \in \hat{G}} d_{\pi}\left|\alpha_{\pi}\right|^{p}<\infty$ while $\sum_{\pi \in \hat{G}} d_{\pi}\left|\alpha_{\pi}\right|^{q}=\infty$. Letting now $T_{\pi}=\alpha_{\pi} P_{\pi}$, where $P_{\pi}$ is a projection on a one-dimensional subspace of $H_{\pi}$, and $T=$

$\oplus_{\pi \in \hat{G}_{\pi}} T_{\pi}^{\left(d_{\pi}\right)}$, we have $T \in \mathcal{S}_{p}(G)$ but $T \notin \mathcal{S}_{q}(G)$. For general locally compact groups the classes $\mathcal{S}_{p}(G)$ may coincide, e.g. in the case of discrete groups, where $\mathcal{S}_{p}(G), p \geq 1$, are all equal to $C_{r}^{*}(G)$.

Acknowledgement We would like to thank the anonymous referee for a number of useful suggestions that led to a substantial improvement of the manuscript.

\section{REFERENCES}

[1] W. B. Arveson, Operator algebras and invariant subspaces, Ann. Math. (2) 100 (1974), 433-532.

[2] J. Bergh And J. LÖFStröm, Interpolation spaces. An introduction, Grundlehren der Mathematischen Wissenschaften, No. 223, Springer-Verlag, 1976.

[3] M. Bożejko And G. Fendler, Herz-Schur multipliers and completely bounded multipliers of the Fourier algebra of a locally compact group, Boll. Un. Mat. Ital. A (6) 2 (1984), no. 2, 297-302.

[4] M. BożEJKo, Sets of uniqueness on noncommutative locally compact groups, Proc. Amer. Math. Soc. 64 (1977), no. 1, 93-96.

[5] M. Boż̇JKo, Sets of uniqueness on noncommutative locally compact groups. II, Colloq. Math. 42 (1979), 39-41.

[6] K. R. Davidson, "Nest Algebras", Pitman Research Notes in Mathematics 191, Longman, 1988.

[7] J. A. Erdos, A. Katavolos and V. S. Shulman, Rank one subspaces of bimodules over maximal abelian selfadjoint algebras, J. Funct. Anal. 157 (1998), no.2, 554-587.

[8] P. Eymard, L'algèbre de Fourier d'un groupe localement compact, Bull. Soc. Math. France 92 (1964), 181-236. 
[9] G. B. Folland, Fourier analysis and its applications, American Mathematical Society, 2009.

[10] J. Froelich, Compact operators, invariant subspaces and spectral synthesis, J. Funct. Analysis 81 (1988), 1-37.

[11] P. Jolissaint, A characterisation of completely bounded multipliers of Fourier algebras, Colloquium Math. 63 (1992) 311-313.

[12] J. Ludwig, N. Spronk And L. Turowska, Beurling-Fourier algebras on compact groups: spectral theory, J. Funct. Anal. 262 (2012), no. 2, 463-499.

[13] J. Ludwig AND L. TuRowskA, On the connection between sets of operator synthesis and sets of spectral synthesis for locally compact groups, J. Funct. Anal. 233 (2006), 206-227.

[14] G. W. Mackey, A theorem of Stone and von Neumann, Duke Math. J. 16 (1949), 313-326.

[15] V. V. PELLER, Hankel operators in the perturbation theory of unitary and selfadjoint operators, Funktsional. Anal. i Prilozhen. 19 (1985), no. 2, 37-51, 96.

[16] A. Pietsch AND H. Triebel, Interpolationstheorie für Banachideale von beschränkten linearen Operatoren, Studia Math. 31 (1968), 95-109.

[17] V. S. Shulman, I. G. Todorov and L. Turowska, Closable multipliers, Integral Equations Operator Theory 69 (2011), no. 1, 29-62.

[18] V. S. Shulman, I. G. Todorov And L. Turowska, Sets of multiplicity and closable multipliers of group algebras, preprint, arXiv:1401.2620, to appear in J. Funct. Anal.

[19] N. Spronk, Measurable Schur multipliers and completely bounded multipliers of the Fourier algebras, Proc. London Math. Soc (3) 89 (2004), 161-192.

[20] N. Spronk And P. Wood, Diagonal type conditions on group $C^{*}$-algebras, Proc. Amer. Math. Soc. 129 (2001), no. 2, 609-616.

[21] E. M. Stein And G. Weiss, Introduction to Fourier analysis on Euclidean spaces, Princeton University Press, 1971.

[22] D. Williams, Crossed products of $C^{*}$-algebras, American Mathematical Society, 2007.

[23] A. Zygmund, Trigonometric series, Cambridge University Press, 1959.

Pure Mathematics Research Centre, Queen's University Belfast, Belfast

BT7 1NN, UNITED KINGDOM

E-mail address: i.todorov@qub.ac.uk

Department of Mathematical Sciences, Chalmers University of Technology and the University of Gothenburg, Gothenburg SE-412 96, Sweden

E-mail address: turowska@chalmers.se 